\title{
A European Roadmap for exoplanets
}

\author{
Artie P. Hatzes ${ }^{1}$ \\ and The Exoplanet Roadmap Advisory Team (EPR-AT)
}

${ }^{1}$ Thüringer Landessternwarte Tautenburg, Sternwarte 5, D-07778, Germany email: artie@tls-tautenburg.de

\begin{abstract}
The Exoplanet Roadmap Advisory Team (EPR-AT) was formed by the European Space Agency (ESA) to advise it on the best path for characterizing exoplanets including terrestrial planets. The EPR-AT delivered its report to ESA in August 2010. Here we summarize the findings of this task force.
\end{abstract}

Keywords. planetary systems

\section{Introduction}

The study of exoplanets is arguably one of the most exciting and vibrant fields in astronomy. Understanding the process of planet formation and why life developed on our Earth provides answers to fundamental questions important to both scientists and the public.

The European Space Agency (ESA) created an advisory panel, The Exoplanet Roadmap Advisory Team (EPR-AT) to advise it on the best roadmap for the characterization of exoplanets up to and including terrestrial planets. This is no easy task as the field is technologically challenging. The detection and characterization of exoplanets requires a diverse range of astronomical measurements that are pushed to the extreme limits: radial velocity precisions of $0.1-1 \mathrm{~ms}^{-1}$, astrometric measurements of a few microarcseconds, photometric variations of $\sim 10^{-5}$, and the measurement of contrast ratios of $\sim 10^{-10}$. The needs of the exoplanet community are drivers of new technologies and many of these extreme measurements often require expensive space missions.

The members of the EPR-AT that drafted the roadmap were Artie Hatzes, (Chair, Thüringer Landessternwarte, Germany), Anthony Boccaletti (Observatoire de Meudon, France), Rudolf Dvorak (Institute for Astronomy, University of Vienna, Austria), Giusi Micela (INAF - Osservatorio Astronomico di Palermo, Italy), Alessandro Morbidelli, Observatoire de la Cote d'Azur, France), Andreas Quirrenbach (Landessternwarte, Heidelberg, Germany), Heike Rauer (German Aerospace Center (DLR), Germany), Franck Selsis (Laboratoire d'Astrophysique de Bordeaux (LAB), France), Giovanna Tinetti, University College London, United Kingdom), and Stephane Udry, University of Geneva, Switzerland).

Space only permits us to summarize our report. The entire roadmap can be downloaded at the ESA website at

http://sci.esa.int/science-e/www/object/index.cfm?fobjectid= 47855 .

\section{The Paths to Characterization}

The study of exoplanets is rapidly moving from one that was dominated by detections, to one where the characterization of exoplanets are producing the most exciting results. The explosion in transiting planet discoveries have enabled us to measure the 
mass, radius, mean density, and dominant atmospheric spectral features of exoplanets. Transiting planets have largely driven this shift to characterization. We are at the point where we can start doing comparative exo-planetology: comparing exoplanets not only to each other, but also to the planets of our own solar system. Drawing from solar system planetary studies, exo-planetary science covers three broad themes: 1) Detections: understanding the census and architecture of planetary systems. 2) Characterization of the internal structure: measuring the fundamental exoplanetary parameters of mass and radius and comparing these to structure models. 3) Characterization of the exoplanetary atmospheres: understanding the effective temperature, composition, and presence of possible biosignatures in the atmospheres of exoplanets.

For these reasons an exoplanet roadmap is not a simple path carrying us from point ' $\mathrm{A}$ ' (the present) to point ' $\mathrm{B}$ ' (the detection of an exo-earth). Rather it is more like a 3-lane road whose lanes are defined by our themes of detection, the characterization of the internal structure, and the characterization of the atmospheres. Each lane moves at its own pace and with developments, both scientific and technological, providing a burst of speed that is often unpredictable. This 3-lane roadmap is schematized in Figure 1. The "Near-term" is defined roughly as the time frame 2011-2017, "Mid-term" 2015-2022, and "Long-term" beyond 2022.

The "fastlane" of exoplanet studies is clearly detections. In the past 15 years approximately 500 exoplanets have been discovered. The "moderate" lane is now held by characterization studies of the internal structure. This progress has largely been fueled by the explosion in transiting planet discoveries now spearheaded by the CoRoT and Kepler space missions. The mass, radius, and mean density has been characterized for over 100 transiting exoplanets. Initial results could only be obtained on giant gaseous planets, but with CoRoT and Kepler we have now been able to characterize the mass, radius, and density of the first rocky planets. The "slowlane" is defined by atmospheric detections. Chemical species such as $\mathrm{CH}_{4}, \mathrm{H}_{2} \mathrm{O}, \mathrm{CO}$ and $\mathrm{CO}_{2}$ have been observed in a handful of exoplanets, all in transiting systems. The progress in the characterizations of exoplanet atmospheres has been hindered by the technological challenges of detecting the tiny $\left(\approx 10^{-5}\right)$ signal of the exoplanetary atmosphere compared to the stellar signal. But these discoveries hints at the future developments in this area once the characterization of exoplanetary atmospheres have reached full maturity.

\section{Key Questions}

The goal of this roadmap is to perform comparative planetology of a wide range of planetary systems including planets down to the terrestrial mass regime in the habitable zone of F-M dwarf stars. Although detections are an important step, the community should strive to make those "added value" discoveries, those which will lead to characterization studies.

The key questions we should answer in this journey of comparative exoplanetology are:

(a) What is the diversity and architecture of exoplanetary systems as a function of stellar parameters and birth environment?

(b) What is the diversity of the internal structure of exoplanets?

(c) What is the diversity of exoplanetary atmospheres?

(d) What is the origin of the diversity and how do planets form?

(e) What are the conditions for planet habitability, how common is exo-life and can we detect the biosignatures. 

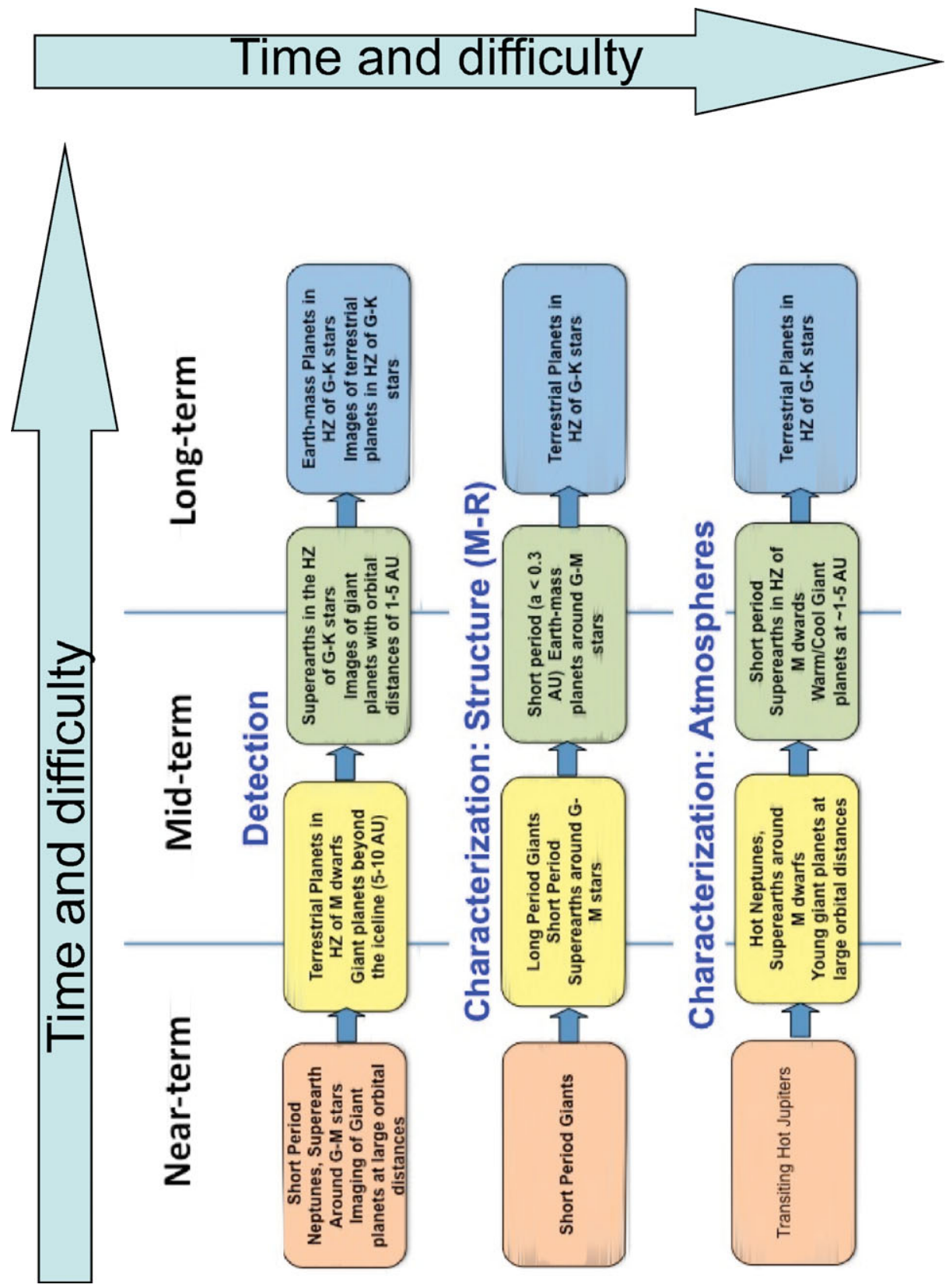

Figure 1. Schematic showing the rough timeline for milestones for our '3-lanes' of detection, characterization of internal structure (mass, radius, mean density) and the characterization of the atmospheres of exoplanets. Near term is approximately 2011-2017, Mid-term 2015-2022, and Long-term beyond 2022. Technological difficulty and time proceeds left to right, and top to bottom. 


\section{Milestones}

As with any roadmap one must have milestones to gauge whether sufficient progress is being made in the field. Some of these milestones include.

Major Goals for Detections:

- A Complete census of exoplanets. If we are to understand planet formation we must obtain a representative census of the types of planets that can form around stars. Detections need to push the parameter space to planets of smaller masses and at larger orbital distances, especially around solar-type stars. It also needs to search for planets around host stars with as wide a range in stellar mass as possible and in different evolutionary states. Currently, the census of known extrasolar planets is heavily biased towards solar type stars. To get this complete census one must use a variety of methods: radial velocity measurements, the most successful detection method to date, astrometric measurements which can obtain the true mass of the exoplant, microlensing surveys as these are one of the few ground-based methods that can detect terrestrial planets in the habitable zone of stars, and direct imaging methods which can detect planets at large orbital distances to the parent stars.

- An Understanding of the Architecture of Planetary Systems. It is probably a good assumption that all planets are found in planetary systems. However, the multiple planet hosting stars is relatively small - about $10 \%$ of the stars known to host at least one exoplanet. The number of known planetary systems must be increased if we are to make progress understanding the architecture of planetary systems.

- Targets for Characterization Discovery of extrasolar planets should only be considered as a first step towards the path of characterization. Detection methods should focus on discoveries for which we can characterize the internal structure (mass, radius, and density) and atmospheric composition. Foremost among these discoveries would be terrestrial planets in the habitable zone of stars, in particular stars like our sun.

Major Milestones for Structure Studies

- The Mass-Radius relationship for Jovian Planets out to 1 AU

- The Mass-Radius relationship for Neptunian Planets out to $1 \mathrm{AU}$

- The Mass-Radius relationship for Terrestrial Planets out to $1 \mathrm{AU}$

The mass-radius relationship is the fundamental quantity that is needed for comparative planetology of the internal structure of exoplanets. Since this requires a radius determination this relationship can only be derived for transiting planets and proceeds from the the easiest (giant planets) to the most difficult (terrestrial planets). Realistically this relationship can only be derived for planets out to $\approx 1$ AU due to the low probability for transits.

Major Goals for Atmospheric Studies

- Composition of Jovian/Neptunian planets out to $0.1 \mathrm{AU}$

- Composition of Jovian/Neptunian planets at greater than 5 AU

- Composition Terrestrial Planets out to $0.1 \mathrm{AU}$

- Composition of terrestrial planets out to 1 AU.

- The detection of biosignatures

The most difficult characterization studies is of the stellar atmospheres and is easiest done for transiting giant planets, giant planets at large orbital distances, transiting terrestrial planets, and finally terrestrial planets in the habitable zone of G-K stars. 


\section{Recommendations}

Our general recommendations are divided between "ground-based", those which are best pursued with ground-based facilities, and "space-based", those which can only be accomplished from space.

\subsection{Detections}

Ground-based

(a) Continued Radial Velocity Searches. The RV has discovered most of the known exoplanets and it will continue to play a dominant role in the forseable future. RV searches will help complete the census of exoplanetary systems out to about 5 AU for G-M stars. It also plays a key role in characterization studies of transiting systems by providing the planet mass. Unfortunately, with the follow up transiting planet candidates, particularly from the CoRoT and Kepler missions, the telescope resources for RV work is overtaxed. The community should ensure that adequate telescope resources are available for carrying out this work, possibly with dedicated 2-4 m telescopes.

(b) Towards a Golden Sample of Stars in the Habitable Zone of G-type stars. With the demise of SIM-Lite the community must now rely on other methods for providing a sample of stars in the habitable zone of solar-type stars. RV measurements should be able to do this given sufficient telescope resources. RV surveys possibly with dedicated telescopes should focus on a relatively small sample of G-type stars $(\approx 50)$. High cadence observations should enable us to "beat down" the intrinsic stellar noise and to detect the $\approx 10 \mathrm{~cm} \mathrm{~s}^{-1}$ amplitude of terrestrial planets. This "Golden Sample" can then serve as the target list for a future flagship mission for characterization studies.

(c) Ground-based microlensing for low mass planets and statistics. Although microlensing does not provide targets for further characterization studies, it does provide important statistics on the lowest mass planets. These studies can be performed from the ground using a network of modest-sized (1-2 m) telescopes.

(d) $R V$ and transits searches for terrestrial planets in the $H Z$ of $M$ dwarfs targets for characterization studies $\mathrm{M}$ dwarf stars may offer us the first opportunity to study the atmospheres of terrestrial planets in the habitable zone of other stars. However, before we can perform characterization studies, either from space or from the ground, targets should first be found.

(e) Imaging of planets at large orbital radii (Planet Finders) The next generation of Planet Finders at the Very Large Telescope will enable us to complete the census of planets out to large orbital radii. Because of the large separation between planet and host stars these systems will be the easiest for studying the exoplanetary atmospheres. Space-based

(a) Use of GAIA to discover a large number of giant exoplanets including determination of mass and orbital inclinations. GAIA has the potential to discover a large number of giant exoplanets and examine such important phenomena such as the misalignment of planetary systems. This mission should be continued as long as possible to increase time base.

(b) Astrometric detection of terrestrial planets in the $H Z$ of solar type stars. Astrometric detections are important because they not only may be more effective at discovering terrestrial planets in the habitable zone of G-K stars, but they also provide us with the true mass - the fundamental parameter of an exoplanet. Because of this the astrometric mission SIM-Lite played a key role in this roadmap. The U.S. Decadal Review did not support SIM-Lite, therefore there is now a gap in ourroadmap in regards to 
astrometric measurements. We hope that in the future a "targeted (as opposed to the global) astrometry can achieve the exoplanet goals of SIM-Lite butat significantly reduced cost.

\subsection{Characterization: Structure}

Ground-based

(a) Continue Ground-based transit studies. Although the space missions CoRoT and Kepler have produced exciting results in the area of transit detections, and hopefully PLATO will do so in the future, the era of ground-based transit searches is not completely over. Ground-based facilities can continue to make contributions by searching for transiting planets in different environments (e.g. stellar clusters) and by searching for photometric transits among the RV-discovered planets, particularly long period planets. Ground-based programs can also do important work by revising the emphemeri of known transiting planets and to search for additional planets via transit timing variations.

(b) Calibration of evolutionary tracks mass and radius of planets detected with imaging at large orbital distances. Direct imaging detections are important as these probe the planet formation at large orbital distances. This method has the disadvantage in that the mass of the planet is poorly determined and must rely on planet evolutionary tracks. These must be calibrated using planets at large orbital distances for which dynamical masses can be derived.

(c) Ground based support for CoRoT and Kepler and preparation of Ground-based support for GAIA and PLATO. CoRoT and Kepler have demonstrated that the ground-based follow-up observations in support of transit missions is enormous. PLATO will further tax these limited resources. The community must ensure that the necessary ground-based resources are in place for these important missions.

$\underline{\text { Space-based }}$

(a) Continuation of CoRoT and Kepler for as long as possible. The exoplanet community is fortunate that it has two working space missions that are producing breakthrough results. The community should ensure that these continue for as long as possible.

(b) Characterization of exoplanets with PLATO. An important milestone of this roadmap is the deriving the mass-radius relationship and internal structure for terrestrial planets as these give important clues as to the formation process. Accurate values of the planet radius and mass are needed to distinguish whether the terrestrial exoplanet has a structure similar to the Earth, more like the Moon, or like Mercury. The measurement of the planet radius requires space-based photometry, while the measurement of its mass means that the targets should be relatively bright. PLATO thus represents a very important, next-step in this roadmap. PLATO will also play a very important secondary role in providing targets for future studies to detect the atmospheres of these transiting terrestrial planets.

\subsection{Characterization: Atmospheres}

\subsubsection{Ground-based}

(a) Characterization of Giant transiting planets with visible and IR facilities

(b) Spectroscopy of planets at large orbital radii using very large and extremely large telescopes 


\subsubsection{Space-based}

(a) Effective use of JWST for characterization studies. The James Webb Space Telescope (JWST) has the capabilities of exoplanet spectral characterization. Although JWST will be a valuable facility for performing characterization studies of exoplanets, it is a general-purpose facility, so it is expected that relatively few targets will be observed. The amount of JWST time available to European scientists will be limited (NIRSPEC is a European contribution and MIRI is a 50-50 US-Europe collaboration). The European community must move fast and organize itself so as to effectively use its share of the time effectively on exoplanet studies. Open Time Key Programs for Exoplanets on JWST, is a concept that worked well with Herschel and should be considered by European scientists in this context.

(b) M-class mission in Cosmic Vision 2 to characterize giant planets and if possible superearths. Efforts to characterise the atmospheres of exoplanets should span the entire parameter space from hot, close in planets, to the cooler ones at large distances that are analogous to what is found in our solar system. In the mid-term roadmap preparations for a mission to perform this characterization should be undertaken. We can identify two classes of exoplanet for which spectral characterization can be realistically done in the near term:

1. Combined light mission: This is aimed at the atmospheric characterisation of hot and warm (including the habitable-zone of M-type stars) Giants, Neptunes, and SuperEarths using transit or combined-light spectroscopy. Spitzer, Hubble and large groundbased observatories have already demonstrated the feasibility of such work. While JWST and ground-based facilities will produce similar results for a select sample of targets, the proposed dedicated mission should provide repeated observations of a much larger sample of stars over a broader wavelength coverage.

2. Angular Resolved Detections. These investigations involve the use of high contrast imaging to minimize the light from the host star and to detect directly the light from the exoplanet. In the midterm such spectral characterizations would be for mature giant planets down to maybe super- Earth size at distances $>1$ AU from the host star.

(c) In the long term (20-25 years) a flagship space mission to characterize terrestrial planets in the HZ of FGK type stars should be flown

Before embarking on such a mission it is essential that the target list be known. This should be a characterization mission and not a discovery mission

\subsection{Technology}

There are a number of technology advancements which are also important for this roadmap:

(a) Improved Wavelength Calibration. This is important for achieving an RV precision of a few $\mathrm{cm} \mathrm{s}^{-1}$.

(b) Detector Development. The greatest need is for mid IR detectors in the range 5-20 $\mu \mathrm{m}$ with improved noise level and stability

(c) Improved Deformable Mirrors that can achieve very small wavefront errors. This is important for angularly resolved detections of exoplanets.

(d) Ongoing developments in cornographic technology

(e) Free flying and deployable structures. These are needed if one is to use external occulters to block the light of host stars. 
It is hoped that in 20-25 years a flagship space mission is flown to characterize terrestrial planets in the habitable zone of G-K stars. This will require the extraction of the feeble signal of the planet that has an intensity that is $10^{-10}$ of the star. Before such a mission is flown the community needs to determine which technology: coronography, nulling interferometry, external occulters, etc. has the highest chance for success. This needs to be done in the next decade otherwise the community risks spreading its limited resources over too many technologies. 